\title{
Semblanza de mis 37 años de servicio en la Cátedra de Bioquímica, el Instituto de Bioquímica y Nutrición y el Centro de Investigación de Bioquímica y Nutrición de la Facultad de Medicina, Universidad Nacional Mayor de San Marcos
}

\author{
Marino Villavicencio-Núñez ${ }^{1}$
}

En el presente artículo, el Dr. Marino Villavicencio Núñez, profesor emérito de la
Universidad Nacional Mayor de San Marcos, narra sus vivencias desde los primeros
pasos en la docencia como profesor Jefe de prácticas a tiempo parcial y los hechos
que dejaron huella en su formación, permitiendo ser considerado como uno de los más
destacados investigadores bioquímicos, con reconocimiento nacional e internacional.
El Dr. Villavicencio es parte y gestor de la historia del Centro de Investigación de
Bioquímica y Nutrición. Quién mejor que él para relatar los detalles de la organización
de los laboratorios, el equipamiento, la capacitación de sus miembros y la producción
científica del más alto nivel, que llevó a que nuestra institución fuera considerada
como un Centro de excelencia en Bioquímica en América Latina. El Dr. Marino
Villavicencio es un auténtico Maestro y, si bien cesó a los 37 años de servicio, sigue
enseñándonos a través de sus libros de bioquímica, sus compendios de actualizaciones
bioquímicas y sus sabios consejos.
Perú - bioquímica, historia; Villavicencio Núñez, Marino; autobiografía; bioquímica,
historia; investigación; OEA.

Biographical sketch of 37 years of service at the Chair of Biochemistry, the Biochemistry and Nutrition Institute, and the Biochemistry and Nutrition Research Center, Faculty of Medicine, Universidad Nacional Mayor de San Marcos

Abstract

Universidad Nacional Mayor de San Marcos' Emeritus Professor Dr. Marino Villavicencio Nuñez narrates in this article his personal experiences starting with early teaching steps as partial time Training chief and facts that traced his formation and national and international acknowledgement as one of the most outstanding researchers in biochemistry. Dr. Villavicencio is part and the manager of Biochemistry and Nutrition Research Center's history. Nobody could relate better laboratories' organization details, equipment, staff capacitation, and the highest level scientific production that lead our institution to be considered as an excellency biochemistry center in Latin America. Dr. Marino Villavicencio is an authentic Master and even though he ceased with 37 years of professional services he continues teaching us through his books on biochemistry, his up to date biochemistry digests and his wise counseling.

1 Profesor Emérito, Centro de Investigación de Bioquímica y Nutrición Alberto Guzmán Barrón. Facultad de Medicina, Universidad Nacional Mayor de San Marcos. Lima, Perú.
Key words: Peru - biochemistry, history; Villavicencio Nuñez, Marino; biochemistry, history; research; OEA.

\section{La Cátedra de Bioquímica}

Cuando estudiaba el primer año de Medicina, en el que se llevaba el curso de bioquímica, la Cátedra de Bioquímica tenía un local muy reducido, ocupaba el primer piso del ala derecha del local central de la Facultad de Medicina. La sala frente a la puerta principal del Decanato era la oficina del titular de la cátedra; la sala siguiente, más grande, era el laboratorio de investigaciones donde se hacía los trabajos de tesis. Los únicos aparatos relevantes eran un fototocolorímetro Klett-Summerson, un fluorómetro y un aparato de Van Slyke. La sala siguiente servía de depósito de material de laboratorio y el pasadizo fue cerrado para implementar un salón para las prácticas de Laboratorio de los estudiantes.

La Cátedra de Bioquímica era una de las mejor organizadas; el Catedrático Titular era el Dr. Alberto Guzmán Barrón, quien había tenido un adiestramiento en investigaciones bioquímicas 
en el laboratorio de su hermano, el Prof. Eleazar Guzmán Barrón, en la Universidad de Chicago. Las clases del Prof. Alberto Guzmán eran muy actualizadas, por su contacto permanente con su hermano y las revistas especializadas que disponía. Su Auxiliar era el Dr. Vitaliano Manrique, quien dictaba el Capítulo de Proteínas. Mi Jefe de Prácticas era el Q.F. Carlos Payva Carbajal, profesional muy estricto y un químico competente. El Ayudante de Cátedra Juan Angulo Bahr, nos hizo algunas prácticas especiales.

A inicios del gobierno del Dr. José Luís Bustamante y Rivero (1945-1948), el Parlamento Nacional aprobó una nueva Ley Universitaria, en la que se incorporaba el tercio estudiantil en los Órganos de Gobierno de la Universidad. Se establecieron las Cátedras Paralelas. En Bioquímica había una Cátedra a cargo del Prof. Alberto Guzmán Barrón y su grupo y otra a cargo de profesores del Instituto de Biología Andina. Con el golpe militar del General Manuel A. Odría (1948), se anuló el Estatuto Universitario y las Cátedras volvieron a su estatus anterior.

En 1950 fui llamado para colaborar en la Cátedra de Bioquímica como Jefe de Prácticas. Los otros Jefes de Práctica eran los Drs. Guillermo del Carpio, Gabriel Gurmendi Robles, Manuel Morante Miranda, Aníbal Llanos, Pedro Martínez y Julio Vílchez. El Titular de la Cátedra era el Dr. Alberto Guzmán Barrón y sus Profesores Auxiliares eran los Drs. Vitaliano Manrique, Juan Angulo Bahr y Carlos Payva Carvajal; entre los Ayudantes de Cátedra recuerdo al estudiante Alberto Cazorla Tálleri.

La dictadura militar hizo sentir su influencia en la Universidad. Es así que, anulado el Estatuto Universitario y la salida del Rectorado del Dr. Luis Alberto Sánchez, se realizaron, en la Universidad de San Marcos, nuevas elecciones. El Dr. Alberto Guzmán Barrón, quien tenía una posición en el Laboratorio Central del Ejército, fue trasladado al Cuzco, por no apoyar la elección de un candidato a Rector que gozaba de la simpatía del gobierno, lo que le obligó a pedir su baja del Ejército. Paralelamente, en la Facultad de Medicina, también hubo cambio de la autoridad y el Dr. Guzmán fue compensado económicamente para que dedicara más tiempo a la Cátedra de Bioquímica.

\section{Gestión de una beca para investigaciones bioquímicas}

También en el año 1950 se realizó en Lima el Congreso Sudamericano de Química, con asistencia del Prof. Eleazar Guzmán Barrón, de la Universidad de Chicago. El Prof. Alberto Guzmán Barrón y algunos integrantes de la Cátedra de Bioquímica participamos en el evento. En esta ocasión, el Prof. Alberto Guzmán nos manifestó que le había hablado a su hermano Eleazar para que fuera a adiestrarme en investigaciones bioquímicas en la Universidad de Chicago.

Como iba trascurriendo el tiempo y no se concretaba la beca de perfeccionamiento, acepté, en 1952, el ofrecimiento del Instituto Nacional de Enfermedades Neoplásicas, para hacerme cargo de la Jefatura del Laboratorio de Bioquímica de dicha institución, conservando mi posición a tiempo parcial en la Cátedra de Bioquímica. Por entonces, el Prof. Eleazar Guzmán Barrón ofreció una conferencia en el Instituto Nacional de Enfermedades Neoplásicas, ocasión en la que el Dr. Eduardo Cáceres Graziani, Director del Instituto, le solicitó que me consiguiera una beca para ir a Chicago a entrenarme con el Dr. Eleazar Guzmán, en investigaciones de Bioquímica del Cáncer. Desde entonces mantuve comunicación directa con el Prof. Eleazar Guzmán Barrón, para informarme del progreso de las gestiones. El Prof. Eleazar Guzmán, atendiendo el pedido del Dr. Cáceres, había solicitado la beca a la Fundación Rockefeller. Yo le comuniqué al Prof. Alberto Guzmán Barrón sobre la posibilidad de esta beca que me ofrecía el Instituto Nacional de Enfermedades Neoplásicas y él me sugirió, actualizando su oferta anterior, que sería mejor que se postulara la beca por la Facultad de Medicina de San Fernando, para que a mi vuelta me reintegrara a la Cátedra de Bioquímica a tiempo integral. Entonces, consulté este problema con su hermano; el Prof. Eleazar Guzmán Barrón y el Dr. Alberto Guzmán eran de la misma opinión, ya que la Cátedra de Bioquímica ofrecía un mejor futuro académico. De allí que, cuando vino a Lima un alto funcionario de la Fundación Rockefeller, a mediados de 1953, las entrevistas que sostuvo fueron con el Dr. Cáceres, el Prof. Alberto Guzmán Barrón, el Decano de la 
Facultad de Medicina Prof. Oswaldo Hercelles y conmigo. Un tiempo después de estas entrevistas recibí de la Fundación Rockefeller los formatos de aplicación para la beca, que debía ser respaldada por la Institución, a la cual me reincorporaría a mi vuelta. Como la decisión ya estaba hecha, se realizaron los trámites con el respaldo de la Facultad de Medicina.

\section{Actividades en uso de la beca otorgada por la Fundación Rockefeller}

En enero de 1954, recibimos la noticia de que la beca me había sido concedida y al mes siguiente viajé a Chicago. Fueron dos años y medio de una fructífera experiencia en el Laboratorio del Prof. Eleazar Guzmán Barrón y otros Centros a donde me envió por períodos cortos: a) al Laboratorio Biológico de Marina de Woods Hole, Massachusetts, para tomar un curso de Fisiología a nivel molecular, de dos meses de duración. Entre los profesores estuvieron los futuros Premios Nóbel James Watson, George Wald y Severo Ochoa y, entre los conferencistas, el premio Nóbel Albert Szent-Gyorgi; b) al Laboratorio Nacional de Brookhaven, New York, donde aprendí las complejas técnicas del uso del $\mathrm{C}^{14}$ en el metabolismo intermediario de los carbohidratos; y, c) al Instituto Rockefeller, para Investigaciones Médicas para aprender la separación de los aminoácidos en columna, una novedad del momento. En la Universidad de Chicago tomé algunos cursos y, además, teníamos Seminarios semanales con el Prof. Eleazar, en los que hacíamos una exposición de revisiones bibliográficas sobre un determinado tema. Publicamos con el Prof Eleazar Guzmán Barrón un trabajo sobre vías metabólicas de la glucosa en células linfáticas y el linfosarcoma $\left({ }^{1,2}\right)$, trabajo a nivel molecular, del que quedó muy satisfecho. Se publicó también un avance sobre la fijación de $\mathrm{CO}_{2}$ en ciertas células normales y tumorales $\left({ }^{3}\right)$.

\section{Gestiones del Prof. Alberto Guzmán Barrón en Lima}

Mientras tanto, en Lima, el Prof. Alberto Guzmán Barrón hizo importantes gestiones para conseguir que la Cátedra de Bioquímica se transformara en Instituto de Bioquímica y Nutrición y, asimismo, hizo aprobar la construcción de un nuevo local para el funcionamiento del Instituto. Otro de sus logros fue la consecución, con el apoyo del Decano de la Facultad de Medicina, Dr. Oswaldo Hercelles, de una donación de US $\$ 63894,00$, de la Fundación Kellogg, para la adquisición de equipos e implementos para los Laboratorios de Prácticas de Bioquímica de los estudiantes y algunos equipos de investigación. Parte de los fondos fueron destinados para una estadía de 6 meses del Prof. Alberto Guzmán Barrón, en la Universidad de Harvard, para que observara los avances en la enseñanza de la Bioquímica y otra parte se destinó al pago de un sueldo complementario al Dr. Guzmán Barrón y a mí, para que trabajáramos a dedicación exclusiva. Este sueldo decrecía año a año, hasta su total reemplazo por la Universidad.

\section{Inauguración del Instituto de Bioquímica y Nutrición de la Facultad de Medicina}

A nuestro regreso a Lima, en agosto de 1956, trabajamos en el Instituto de Medicina Social hasta fin de año, en que nos trasladamos al nuevo edificio del Instituto de Bioquímica y Nutrición. La ceremonia de inauguración del Instituto fue el 23 de marzo de 1957, con la asistencia del Dr. Hastings, Profesor de Bioquímica de la Universidad de Harvard $\left({ }^{4}\right)$, y el entonces Decano de la Facultad de Medicina, Dr. Alberto Hurtado.

El nuevo edificio del Instituto de Bioquímica y Nutrición contaba con todas las facilidades para la docencia y la investigación. En el primer piso, había dos grandes aulas acondicionadas para las prácticas de laboratorio de los estudiantes de pregrado. En el segundo piso, oficinas administrativas, una biblioteca, varios laboratorios pequeños, un ambiente para depósito y un cuarto frío. En el tercer piso, tres grandes laboratorios de investigación, con oficinas y cuartos para la instalación de equipos de investigación. En el sótano: un laboratorio para prácticas de los estudiantes y servicios higiénicos. En este último, se instaló ulteriormente un cuarto frío moderno importado, con fondos de la OEA, y se acondicionó los laboratorios de Química Bioorgánica, al firmarse el convenio con la Academia de Ciencias de la Unión Soviética. 


\section{Equipamiento de los laboratorios de investigación}

La Fundación Rockefeller otorgó un donativo de \$9 000,00 al Instituto de Bioquímica y Nutrición, para las Investigaciones de Metabolismo Celular que estaban a mi cargo. Con estos fondos se adquirió un espectrofotómetro Beckman, un aparato de Warburg para medir la respiración celular, una centrífuga refrigerada con su aditamento de multivelocidad y material de laboratorio. Un contador de isótopos radioactivos para trabajos con $\mathrm{C}^{14}$ y un aparato de electroforesis de celda (Tipo Tiselius) fueron adquiridos con los fondos donados por la Fundación Kellogg. La Fundación Rockefeller continuó aportándonos fondos para investigación durante los tres años subsiguientes. Con las donaciones de la OEA (Proyecto Multinacional de Bioquímica) y la donación de la Fundación Kellogg para el Programa de Maestrías en Bioquímica y Fisiología conseguidas ulteriormente, se fue completando el equipamiento con dos espectrofotómetros dotados de registradores, un aparato de cromatografía líquida de alta performance (HPLC), un aparato para cromatografía gas-líquido y otros aparatos menores. Durante el Decanato del Prof. Alberto Guzmán Barrón, la Facultad de Medicina compró una ultracentrífuga preparativa Beckman, para nuestros laboratorios de investigación, y años después se adquirió otra ultracentrífuga preparativa más moderna, con fondos de la OEA. Los fondos de la OEA nos permitieron también mantener nuestras suscripciones en las revistas de la especialidad.

\section{El personal inicial de Docencia e Investigación del Instituto de Bioquímica y Nutrición}

El personal de investigación del Instituto de Bioquímica y Nutrición estaba constituido por el Prof. Alberto Guzmán Barrón y el Q.F. Carlos Payva Carvajal, que se ocupaban del Área de Nutrición. El Dr. Leonidas Delgado Butrón colaboraba con el Dr. Guzmán Barrón, pero más se ocupaba de temas relacionados con la clínica. Posteriormente, la Q.F. Teresa Blanco se adicionó al grupo para trabajar con el Dr. Payva. Yo me trasladé al tercer piso para organizar el equipo de investigación de Bioquímica Básica, con la cooperación de la Q.F. Rosa Guerra, quien fue nombrada Asistente de Investigación. Los Drs. Fermín Rosales Zapata y Arturo Olivera, Jefes de Práctica a Tiempo Completo, de inmediato se asimilaron al grupo, así como el Ayudante de Cátedra Ernesto Melgar Salmón, quien era un brillante estudiante de Medicina. Para la enseñanza, había también profesores a Tiempo Parcial indicados más arriba, de los que, al crearse el Instituto, quedaron: el Dr. Vitaliano Manrique, Profesor Asociado, los Profesores Auxiliares Juan Angulo Bahr, Gabriel Gurmendi Robles, Carlos Payva, Aníbal Llanos, Pedro Martínez y Julio Vílchez. Eran Ayudantes de Cátedra, además, los estudiantes de Medicina José Gutiérrez Correa, Ernesto Melgar Salmón, Guillermo Cremer Nicoli y José Goldman Furman.

\section{El adiestramiento en bioquímica básica}

Me encargué de adiestrar al grupo que trabajaba conmigo en el uso de las técnicas utilizadas en las investigaciones de Bioquímica Básica y teníamos seminarios semanales sobre temas especiales de Bioquímica, para que la preparación experimental se complementara con una buena base teórica. Se trabajó con un entusiasmo febril, destacándose la Dra Rosa Guerra por su habilidad para el trabajo químico, convirtiéndose en la colaboradora obligada en la mayor parte de los trabajos sobre metabolismo intermediario. Se concluyó el primer trabajo de Bioquímica a nivel molecular: el metabolismo de la glucosa en la corteza cerebral. Coincidentemente, recibí una carta del Dr. Francisco Ghiretti, con quién trabajamos juntos en Chicago, pidiéndome un trabajo para publicar en Acta Fisiológica Latinoamericana, en memoria de nuestro común maestro el Prof. Eleazar Guzmán Barrón, fallecido en 1957. Nuestro trabajo fue publicado $\left(^{5}\right)$. Inmediatamente abordamos otro tema: metabolismo de la ribosa-5-fosfato en tejidos de mamíferos, que yo había empezado en Chicago. Concluido, lo enviamos para su publicación a una revista acreditada internacionalmente, Biochimica et Biophysica Acta, y fue aceptado $\left({ }^{6}\right)$. Luego siguieron los trabajos de tesis de Doctorado de Rosa Guerra $\left({ }^{7}\right)$ y de Bachiller de José Goldman $\left({ }^{8}\right)$, que fueron publicados en la misma revista, a nombre de los miembros del equipo que participaron en los trabajos. 
Nuestro estilo de trabajo era de tipo americano, continuo, con un pequeño intervalo a medio día para el refrigerio.

\section{El conflicto en la Facultad de Medicina y elección de un nuevo Decano}

A inicios de 1961, se produjo un grave conflicto en la Facultad de Medicina. El Parlamento Nacional aprobó un nuevo Estatuto Universitario, en el que se establecía la participación del tercio estudiantil en los Órganos de Gobierno de la Universidad; pero, por influencia de las autoridades de la Facultad de Medicina de San Marcos, se adicionó un artículo que establecía que las Facultades de Medicina, por la índole de sus estudios, se regirían en lo académico, administrativo y en la constitución de su gobierno por los dispositivos que dictaran para el efecto dichas Facultades. El Rector Luis Alberto Sánchez, de la Universidad de San Marcos, convocó la Asamblea Universitaria, para hacer extensivo el tercio estudiantil a la Facultad de Medicina. La aprobación de la propuesta provocó la renuncia masiva de los profesores de la Facultad de Medicina. Los profesores fuimos llamados para firmar la renuncia, que fue publicada en los diarios de la capital. El Prof. Alberto Guzmán Barrón viajó en esos días a Moscú, a un congreso de Bioquímica, y aprovechó para visitar diferentes centros de Europa; quedándome yo como Profesor Asociado a cargo del Instituto de Bioquímica y Nutrición. El otro Profesor asociado, el Dr. Vitaliano Manrique renunció, pero como era Profesor Titular de Bioquímica en la Facultad de Odontología, continuó en San Marcos y los profesores a tiempos parciales se apartaron. Con el pequeño núcleo de profesores a Dedicación Exclusiva que trabajaban conmigo, decidimos quedarnos en San Marcos; lo mismo ocurrió con la mayor parte de los investigadores del Instituto de Biología Andina. En total, nos quedamos en la Facultad de Medicina de San Fernando alrededor de $20 \%$ de los profesores. Fui llamado por el Rector Sánchez para integrar la Junta Transitoria de Gobierno de la Facultad de Medicina. Pedí un tiempo prudencial para decidirlo, ya que el Director del Instituto estaba ausente y terminé incorporándome a la Junta Transitoria, en setiembre de 1961. Al regresar el Prof. Guzmán Barrón lo mantuvimos un tiempo en su cuartel de invierno antes de su incorporación a la Junta
Transitoria. Se realizó un concurso y casi todos los Profesores Asociados ascendieron a Principales. Yo estaba impedido de ascender, porque la organización arcaica, de tipo europeo, de nuestra Universidad no permitía sino un Profesor Principal por Cátedra. Se formó un Consejo de Facultad que, en 1962, eligió como Decano de la Facultad de Medicina al Prof. Alberto Guzmán Barrón. En este mismo año, obtuve mi Doctorado en Medicina.

\section{Actividades en mi segundo viaje a los Estados Unidos de Norteamérica}

Previniendo los acontecimientos, ya mucho antes del conflicto, había hecho gestiones para volver a los Estados Unidos por un tiempo. Me ofrecieron una posición en la Universidad de Syracuse y conseguí una beca de la John Simmon Guggenheim Memorial Foundation, una beca muy honorífica que proporcionan al investigador para que trabaje en el sitio que escoja. Opté por la beca de la Guggenheim y, a fines de 1962, me trasladé con mi familia a Detroit para trabajar con Thomas P. Singer, en transporte de electrones, en el Instituto Edsel Ford para Investigaciones Médicas, y el año siguiente en el Departamento de Bioquímica de la Universidad de California (Berkeley), con el Prof. J.B. Neilands, en el campo de la enzimología. En Detroit, colaboré en un trabajo, publicado en Biochem Zeitschrift $\left({ }^{9}\right)$, en memoria al 80 aniversario de Warburg, y en Berkeley publicamos en la revista Biochemistry un trabajo sobre una enzima inducible que degradaba un péptido cíclico, el ferricromo A $\left({ }^{10}\right)$.

Otro de los propósitos de mi segundo viaje a los Estados Unidos de Norte América fue buscar centros de adiestramiento para mis colaboradores del Instituto de Bioquímica y Nutrición, en otras áreas de la Bioquímica, a fin de que se formaran nuevos grupos de investigación. Las universidades americanas atravesaban por una época de bonanza y disponían de fondos para contratar investigadores extranjeros. En nuestra visita a la Universidad de Cleveland, Ohio, se concretó una posición para el recién egresado de la Facultad de Medicina, el Dr. Ernesto Melgar Salmón, quien pasó 2 años (1964-1966) de adiestramiento en investigaciones en ácidos nucleicos y fue quien introdujo en el Perú las investigaciones en biología molecular $\left({ }^{11,12}\right)$. 
En Cleveland, se consiguió también una posición para el adiestramiento en investigaciones del Dr. Fermín Rosales, quién trabajó con el Dr. Sakami $\left.{ }^{(13}\right)$. Visité también el Departamento de Bioquímica de las universidades de Chicago; Tulane, New Orleans; Washington, Saint Louis, Missouri; Colorado, Denver; California, Los Ángeles; Stanford, Palo Alto, California, y Washington, Seattle. En estas universidades investigué la organización de los programas de posgrado en bioquímica, que me sirvieron para estructurar el primer Programa de Maestrías que se organizó en San Marcos: las Maestrías en Bioquímica y Fisiología. En la Universidad de Chicago, obtuve el ofrecimiento del Prof. Donald Steiner, quien investigaba la bioquímica de la diabetes mellitus, de hacer lo posible para que el Dr. Guillermo Cremer, recién egresado de San Fernando, fuera a trabajar con él. El Dr. Cremer prefirió la medicina y se fue a la Clínica Mayo. Unos años después, el Prof. Steiner fue consagrado con el Premio Nóbel en Medicina, por sus aportes en la bioquímica de la diabetes. El Prof. Dille, Jefe del Dpto. de Farmacología de la Universidad de Washington, Seattle, se interesó mucho en establecer nexos con San Marcos. Nos invitó a visitar su Departamento y aún viajó a Lima para concretar un intercambio con el Dpto. de Farmacología, pero, desafortunadamente, no hubo interés del Decanato.

\section{Mi retorno al Perú y algunos cambios en la Facultad de Medicina}

Regresé con mi familia a Lima, en octubre de 1964, último año del decanato del Prof. Alberto Guzmán Barrón. El año siguiente fue elegido Decano el Dr. Jorge Campos Rey de Castro y el Dr Alberto Guzmán Barrón volvió a tomar la Jefatura del Departamento de Ciencias Dinámicas (Bioquímica, Fisiología y Farmacología) y la Dirección del Instituto de Bioquímica y Nutrición. Aquí surgió en la Facultad de Medicina una iniciativa de los Profesores Principales para que se realizara un nuevo concurso para la promoción de profesores. Se logró la creación de una vacante de Profesor Principal de Bioquímica y otra en Fisiología, entre otras plazas. Así, en 1966, fui promovido a Profesor Principal de Bioquímica y el Dr. Andrés Rotta Oliveros a Profesor Principal de Fisiología. Como la Jefatura del Departamento era rotativa, el Dr. Andrés Rotta se hizo cargo, por elección, del Departamento de Ciencias Dinámicas, hasta 1968, en que me tocó el turno de asumir la Jefatura. Cabe resaltar que durante el primer gobierno del Arq. Fernando Belaúnde Terry (1963-1968), los profesores universitarios, especialmente los de Dedicación Exclusiva, estuvimos muy bien remunerados.

\section{El golpe militar, un nuevo Estatuto Universitario y reorganización total de la Universidad}

En octubre de 1968, se produce un golpe militar encabezado por el General Juan Velasco Alvarado. Todos los Ministerios y puestos importantes del gobierno estaban a cargo de militares.

Se promulgó un nuevo Estatuto Universitario, que implicó un cambio radical en la organización de la Universidad. Se creó un Consejo Ejecutivo integrado por el Rector, dos Vice-Rectores y las Direcciones Universitarias; desaparecieron las Facultades, para ser reemplazadas por los Programas Académicos, con funciones limitadas; desaparecieron los Departamentos de las Facultades y se creó los grandes Departamentos, al servicio de todos los Programas Académicos de la Universidad. Se creó el Departamento de Bioquímica y Fisiología para agrupar a cerca de 60 profesores de todas las cátedras (asignaturas) similares de la Universidad. Luego, se realizaron elecciones para nombrar al Rector, Vice-Rectores, Directores de Programa, Jefes de Departamento, Coordinadores de Sección y un delegado a los Programas Académicos. Fui elegido Jefe del Departamento de Bioquímica y Fisiología y el Dr Alberto Guzmán Barrón, Coordinador de la Sección Bioquímica y Delegado del Departamento al Programa Académico de Medicina Humana. En este último, se realizaron elecciones para Director del Programa, habiendo sido elegido el Dr. Alberto Guzmán Barrón. El Rector elegido fue el Dr. Juan de Dios Guevara; Vice-Rector Académico el Dr. Alberto Escobar y Vice-Rector Administrativo el Dr. Alberto Cuba Caparó.

Un avance que trajo el nuevo Estatuto Universitario fue que se acababa con el sistema anticuado de un solo profesor principal por cátedra. Con el 
nuevo estatuto, todo profesor que reunía los requisitos para ascender a una categoría dada tenía derecho al ascenso, incluso a la de Profesor Principal. Desafortunadamente, hubo ulteriormente un relajo en las exigencias, dándose mucha facilidad a los ascensos.

La sede del Departamento de Bioquímica y Fisiología era el Instituto de Bioquímica y Nutrición. El Instituto dependía del Departamento. La departamentalización permitió la centralización de las investigaciones y muy valiosas adquisiciones de nuevos investigadores. Las profesoras Beatriz Lizárraga y Rosa Vásquez Gallardo, excelentes investigadoras de la ex-Facultad de Ciencias Biológicas y que habían obtenido una maestría en Bioquímica en la Universidad de Indiana, Bloomington, Estados Unidos de NA, con beca de la Organización de los Estados Americanos, se incorporaron a nuestro Instituto: la Profesora Lizárraga, para integrar el equipo de Biología Molecular del Dr. Ernesto Melgar, y la profesora Rosa Vásquez, nuestro equipo de Estudios de Metabolismo Celular.

En 1969, el Gobierno Militar creó el Consejo Nacional de Investigación, que tenía un Presidente y varios miembros de diferentes áreas. Yo fui designado para integrar este Consejo, que estaba presidido por el Ing. Alberto Giesecke Matto. Esta feliz circunstancia me permitió vincularme con la Organización de los Estados Americanos, para conseguir los donativos que mencionaré más adelante. El Consejo cesó en sus funciones en 1971 $y$ fue designado un nuevo Presidente.

El Prof. Alberto Guzmán Barrón, aburrido con la poca labor que había que hacer en la Dirección de Programa Académico de Medicina Humana, renunció al cargo y pidió su cese de la Universidad, en 1970, y así yo asumí la conducción del Departamento de Bioquímica y Fisiología y del Instituto de Bioquímica y Nutrición, que dependía del Departamento.

\section{Creación de las Maestrías en Bioquímica y Fisiología}

Al regresar de mi segundo viaje a los Estados Unidos, a fines de 1964, traté de conectarme con la Fundación Kellog, para presentarle un proyecto para la creación de un Programa de Maestrías en Bioquímica y Fisiología, proyecto que se fue estructurando a lo largo de los años 1967 y 1968. En 1969, por fin recibí de la Fundación referida una promesa de estudiar el proyecto. En estas gestiones recibí un respaldo decisivo del Rector de San Marcos, el Dr. Juan de Dios Guevara, quien no solo en su condición de Rector de San Marcos sino también de Presidente del Consejo Nacional de la Universidad Peruana, se dirigió a la Fundación Kellogg respaldando mi proyecto. En marzo de 1971, la Kellogg aprobó un donativo de US\$129600,00 para la creación y funcionamiento de las Maestrías en Bioquímica y Fisiología, por un período de tres años. Los fondos eran para becas de estudiantes y equipos de investigación para Bioquímica y Fisiología. Los fondos para becas eran enviados a la Universidad y los destinados para equipos fueron administrados por la Panamerican Health and Education Foundation, a la que hacíamos llegar las listas de pedidos. El Director de los Programas Académicos de Perfeccionamiento me designó Presidente del Comité de Maestrías en Bioquímica y Fisiología y Coordinador del área de Bioquímica y al Prof. Augusto Vallenas, Coordinador de Área de Fisiología. Nuestro programa fue el primer Programa de Maestrías que se creaba en San Marcos.

\section{El Centro responsable en el Perú del Proyecto Multinacional de Bioquímica de la Organización de los Estados Americanos}

En 1970, había conseguido de la Organización de los Estados Americanos una 'Acción de Refuerzo' de US\$ 21355 para investigaciones bioquímicas y, en 1971, el Programa Regional de Desarrollo Científico y Tecnológico de la OEA estaba apoyando Centros Multinacionales de Adiestramiento en Bioquímica en Brasil, México, Argentina y Chile. Con nuestro Programa de Maestrías en marcha, gestioné ante la OEA que nuestro Centro (Departamento de Bioquímica y Fisiología-Instituto de Bioquímica y Nutrición) fuera reconocido como Centro de Adiestramiento en el Perú del Proyecto Multinacional de Bioquímica de la Organización de los Estados Americanos. Este Proyecto fue apro- 
bado, lo cual implicaba el aporte anual de US\$20 000,00 a $\$ 30000,00$, que se renovaba periódicamente, para equipos, material bibliográfico, contratación de profesores visitantes para el desarrollo de cursos nacionales e internacionales y asistencia de profesores de nuestro centro a congresos nacionales e internacionales. Los fondos donados eran enteramente administrados por la Oficina de la OEA en Lima. La asistencia de la OEA, como Proyecto Multinacional de Bioquímica, se extendió hasta 1979 y ulteriormente como 'Bioquímica para el Desarrollo' continuó aún después de mi cese en la Universidad, en 1987. El apoyo de la OEA fue la contribución más importante para la continuidad del Programa de Maestrias y el desarrollo de las investigaciones en nuestro Centro. El 12 de noviembre de 1986, se realizó la ceremonia de entrega a nuestro Centro como donativo de todos los equipos y material bibliográfico adquiridos con fondos de la OEA hasta ese año, cuyo valor ascendía a unos US\$400 000,00. El Programa de Maestrías, durante mi gestión, permitió preparar alrededor de 100 profesores de Bioquímica y Fisiología, para las universidades del Perú.

Como el cargo de Jefe de Departamento era por rotación, en 1976, cesé como Jefe del Departamento de Bioquímica y Fisiología y quedé con la Dirección del Instituto de Bioquímica y Nutrición, que dependía de la Jefatura del Departamento. Para evitar interferencias y una mayor independencia, solicité que el Consejo Ejecutivo aprobara la transformación del Instituto de Bioquímica y Nutrición en Centro de Investigación de Bioquímica y Nutrición, que por su carácter multidepartamental gozaba de total independencia. Mi solicitud fue aprobada.

\section{Gestiones a favor de los Docentes Universitarios}

Durante la dictadura militar de Velasco Alvarado-Morales Bermúdez hubo un deterioro creciente de las remuneraciones del profesor universitario, muy particularmente de los profesores a dedicación exclusiva, poniendo en peligro el desarrollo de las investigaciones, por la fuga de talentos. Para contrarrestar esta situación creamos en la Universidad Nacional Mayor de San Marcos la Asociación de Docentes a Dedicación Exclusiva y, en cooperación con las otras Asociaciones de Docentes de San Marcos, se eligió un Presidente que representara a la Universidad de San Marcos, función que recayó en mi persona. En coordinación con los Presidentes de las Asociaciones de Docentes de las universidades La Agraria, la Universidad Federico Villarreal y la Universidad Cayetano Heredia, hicimos diversas gestiones. Hablamos con el Ministro de Educación y con el de Economía y Finanzas, consiguiéndose algunos paliativos, pero ninguna mejora substancial de la situación económica del profesor universitario.

Refuerzo de los grupos de investigación con los egresados del Programa de Maestría en Bioquímica y la incorporación de nuevos investigadores

Los grupos de investigación del Centro de Investigaciones de Bioquímica y Nutrición (CIBN) se reforzaron con los egresados de la Maestría en Bioquímica. Los Magísteres Jorge Huamán, Raquel Oré, Alvaro Marcelo e Inés Arnao Salas, quienes hicieron excelentes trabajos de tesis de graduación, se incorporaron a nuestro grupo de trabajo, en el que los integrantes más antiguos éramos yo y las Dras. Rosa Vásquez y Rosa Guerra. Se realizó múltiples trabajos sobre metabolismo de la glucosa en tejidos de alpaca y aislamiento y propiedades de las enzimas glucolíticas y gluconeogénicas en hígado de alpaca $\left({ }^{14,15}\right)$ y algunos trabajos sobre hormona de crecimiento de alpaca, en cooperación con el grupo del Prof. J.A. Santomé, de la Universidad de Buenos Aires $\left({ }^{16,17}\right)$. Los trabajos fueron presentados en Congresos nacionales e internacionales, algunos publicados en revistas internacionales y muchos quedaron por publicarse in extenso.

Las Magísteres Doris Sánchez, Patricia Woll y la Dra. Aura Gil integraron al equipo del Dr. Ernesto Melgar. Otro integrante de este grupo era la investigadora Dra. Beatriz Lizárraga. Este grupo, que trabajaba en Biología Molecular, fue uno de los grupos más productivos del Centro de Investigación de Bioquímica y Nutrición. Sus trabajos sobre desoxirribonucleasa pancreática 1 , la interacción de ADN con las proteínas histonas y la determinación de la estructura de la cromatina en moluscos y cangrejos marinos fueron presentados 


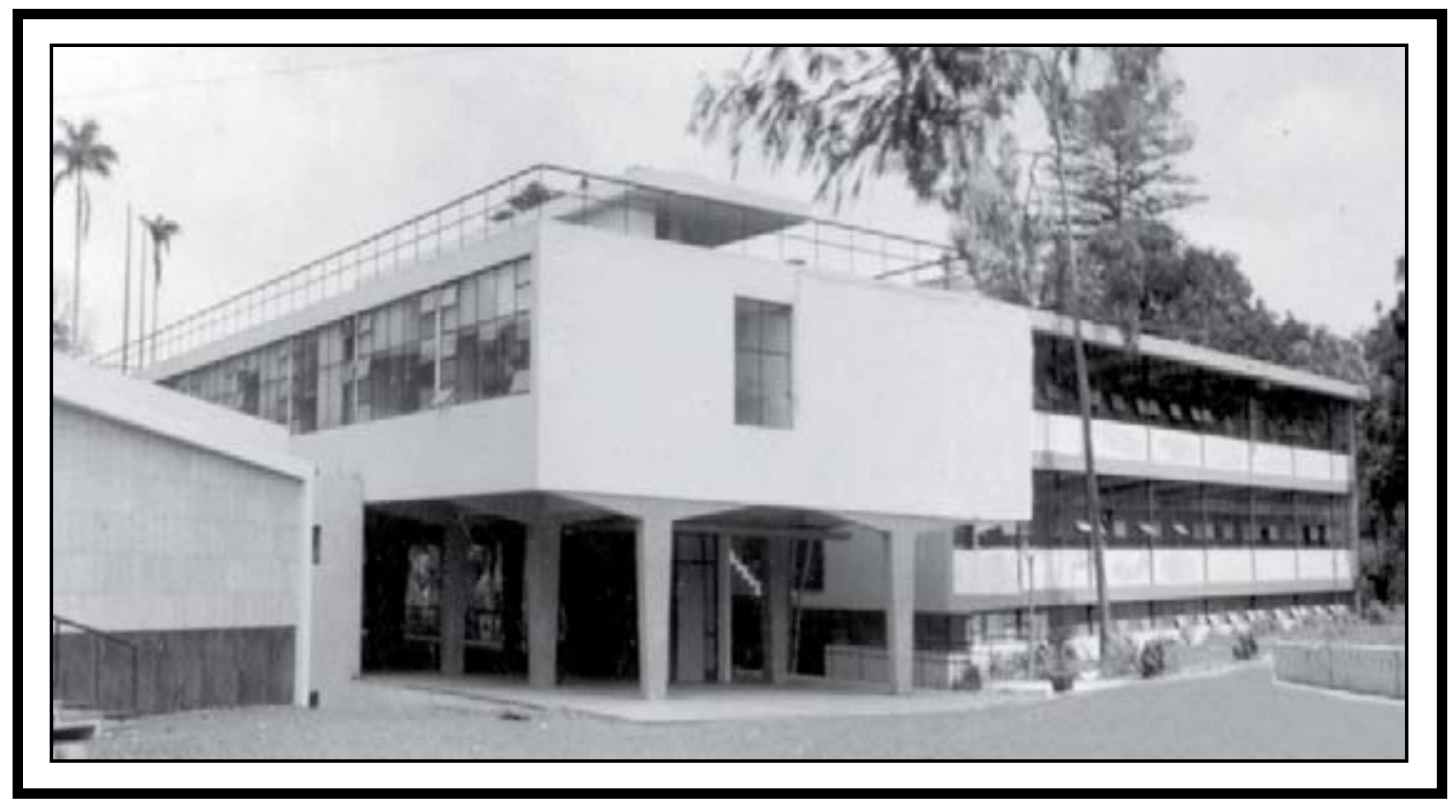

Figura 1. Edificio del Centro de Investigación de Bioquímica y Nutrición Alberto Guzmán Barrón, construido, siendo decano de la Facultad de Medicina el Dr. Oswaldo Hercelles, e inaugurado, el 23 de marzo de 1957, siendo Decano el Dr. Alberto Hurtado.

en congresos nacionales e internacionales y publicados en las revistas internacionales más acreditadas, como J Biological Chemistry, Biochem Biophys Acta $\left({ }^{18-20}\right)$. El Dr. Ernesto Melgar fue, además, distinguido con el premio 'Francisco Camino' a la mejor tesis de Bachiller, en 1962, y con el premio 'Hipólito Unánue' a la investigación científica, en 1982. Fue Profesor Visitante de varias Universidades del Perú. Formó parte de diversas Comisiones Nacionales e Internacionales, entre ellas, miembro del Jurado Internacional para adjudicar el premio 'Bernardo Houssay' de la OEA (1983), miembro del Directorio del Concytec (1980-1985), Jefe del Departamento de Bioquímica y Fisiología (19821983) y Vice-Rector Académico de la Universidad Nacional Mayor de San Marcos (1985-1987). La Dra. Beatriz Lizárraga fue también distinguida con una mención honrosa del premio Hipólito Unánue al mejor trabajo en Bioquímica (1978); en 1985, fue nombrada Directora de la Escuela de Posgrado de la Universidad de San Marcos. Es miembro de diversas sociedades internacionales, entre las que cabe destacar la American Society of Biochemistry and Molecular Biology y la Human Genome Organization. Posteriormente, ha dictado conferencias sobre sus investigaciones en varios países del continente y ha formado parte de importantes Comités Nacionales e Internacionales

Los Drs. Fermín Rosales, Emilio Guija y el Magíster Hielke Haak Mares formaron otro grupo para investigar la fosfatasa ácida en hígado de bovino y alpaca, realizando varias publicaciones $\left({ }^{21,22}\right)$. La profesora Liliana Ballivián, quien se inició con nosotros colaborando en el área de proteínas, consiguió una beca de la ICRO para trabajar con el Prof. Grosjean en la Universidad Libre de Bruselas, en el área de Biología Molecular. $\mathrm{Su}$ inquietud de superación científica la condujo a buscar otras oportunidades de adquirir nuevas experiencias. Es así que consiguió una beca para trabajar con el Prof. Chantrenne, en el Laboratorio de Bioquímica y Biología Celular de la Universidad de Bordeaux, Francia (1976). Desafortunadamente, cuando ya estaba en viaje de vuelta al Perú, murió en un accidente en Londres. El Magíster Mario Monteghirfo, a su retorno de su adiestramiento en los Estados Unidos de NA, abrió una nueva línea de trabajo en biología molecular, con resultados muy productivos. 
La Sección Nutrición de nuestro Centro hizo valiosas adquisiciones. El Dr. Guido Ayala Macedo se incorporó al Centro, en 1970. Tuvo un adiestramiento de un año (1971) en la Sección Biomédica de la División de Crecimiento y Desarrollo del Instituto de Nutrición de Centro América y Panamá (INCAP-Guatemala). Luego, siguió estudios en el Programa de Maestría en Bioquímica y Nutrición en el INCAP y la Universidad San Carlos de Guatemala (1972-1973), graduándose de Magíster en Bioquímica y Nutrición. El Dr. Ayala es coautor del libro 'Nutrición Humana', en dos tomos, con A. Guzmán Barrón y T. Blanco ( $1^{\text {a }}$ edición 1981 y $2^{\text {a }}$ edición 1986) $\left({ }^{23}\right)$, que merecieron el Premio Hipólito Unanue (1987), y ha realizado trabajos de nutrición a nivel nacional de gran valía, incluyendo libros apoyados por organismos internacionales, como la FAO $\left({ }^{24}\right)$. El Dr Ayala es cogestor de la creación de la Escuela Académico Profesional de Nutrición Humana, en la Universidad Nacional Mayor de San Marcos (1976), gestor de la creación del Programa de Maestría en Nutrición, UNMSM, y nombrado Coordinador de la misma, en 1983. Tulio Aguilar Fajardo, profesor de la Universidad Agraria, que había obtenido su Ph.D. en Nutrición, en los Estados Unidos, se incorporó a nuestro Centro, en 1978, e hizo varias publicaciones importantes sobre la metionina. Desafortunadamente, se apartó de la Universidad por motivos de salud. El Profesor Roger Ramos Aliaga, Doctor en Farmacia y Bioquímica, se incorporó a nuestro Centro en 1972. Hizo estudios de posgrado en Nutrición en la Universidad de San Carlos y el Instituto de Nutrición de Centro América y Panamá (INCAP-Guatemala) (1967) e investigaciones en Bioquímica y Neuroquímica en el Centro Nuclear de Puerto Rico (1967-1971), en las Universidades de Chile y Católica de Chile y el Instituto Max-Plank de Alemania Federal, con becas AEC, UPR, PNUD/UNESCO y la Sociedad Max Plank. Realizó numerosas publicaciones en revistas nacionales e internacionales $\left({ }^{25-28}\right)$. En 1982, fue distinguido con el Premio Hipólito Unánue de investigación científica. Sus investigaciones versaron sobre el metabolismo de las drogas, en particular de la cocaina, y el valor biológico de la dieta en el Perú.

Las investigaciones del Prof. Alberto Guzmán Barrón fueron en el área de la Nutrición, en par- ticular sobre las anemias por desnutrición en el Perú y la evaluación del valor biológico de las proteínas, entre otros temas. Algunos trabajos fueron en asociación con el Dr. Carlos Payva y Teresa Blanco, otros con el Dr. Guido Ayala y el Dr. Leonidas Delgado. Publicó un libro sobre Nutrición Humana, en dos tomos ( $1^{\text {a }}$ edición 1981 y $2^{a}$ edición 1986), en colaboración con Teresa Blanco y Guido Ayala Macedo $\left({ }^{23}\right)$.

El Centro de Investigación de Bioquímica y Nutrición tenía también una Sección de Bioquímica Farmacológica, encabezada por el Prof. Aurelio Ortiz e integrada por las profesoras Teresa Núñez e Hilda Jurupe, del Departamento de Farmacología. Hicieron estudios farmacológicos y bioquímicos de los efectos depresores de la cocaína, modificaciones cerebrales de aminoácidos durante la inducción de dependencia alcohólica, la influencia de las modificaciones del metabolismo de los neurotrasmisores sobre el síndrome de abstinencia alcohólica, diversos estudios sobre convulsiones inducidas por pentilenotetrazol, entre otros temas.

Finalmente, había también una Sección de Bioquímica Clínica integrada por los profesores Leonidas Delgado Butrón y Manuel Taboada Vega. Este último profesor siguió estudios en nuestro Programa de Maestría en Bioquímica. Los trabajos que hacían eran generalmente en cooperación con los profesores de la Sección Nutrición.

\section{Becas}

En cuanto a las becas, al Magister Jorge Huamán, de nuestro Centro, se le consiguió una beca para hacer sus estudios de Doctorado en la Universidad de Cambridge (Inglaterra), pero un compromiso antelado lo obligó a renunciar a la beca para hacerse cargo de la enseñanza de Bioquímica en la Universidad de Piura. Al profesor Emilio Guija se le consiguió una posición en la Universidad de Sao Paulo, para trabajar con el Prof. H. Chaimovich, donde obtuvo su Magíster en Bioquímica y retornó a nuestro Centro. El profesor Guido Ayala consiguió una beca de la OPS /OMS para realizar sus estudios de Maestría en la ciudad de Guatemala (INCAP- Universidad San Carlos). Igualmente, las profesoras Inés Arnao, Doris Sánchez y Raquel 
Oré hicieron cortas estadías en el laboratorio del Dr. J.A. Santomé, en Buenos Aires, participando en una investigación conjunta sobre la hormona de crecimiento de la alpaca, que resultó en varias publicaciones en revistas internacionales. El Proyecto fue elaborado por Doris Sánchez, con el grupo del Dr. Santomé y apoyado por el Programa PNUD/UNESCO.

Varios profesores del Centro consiguieron por propias gestiones apoyo para cursos o estadías en el exterior. Así, el Prof. Ernesto Melgar, después de su retorno de los Estados Unidos, consiguió apoyo de la International Agency for Atomic Energy (IAAE), para un curso de Radiobiología realizado en Soeq Nuclear Center, Israel, en 1967. Otra beca, de la misma (IAAE), para un curso sobre efectos biológicos de las radiaciones, realizado en Bahba Atomic Nuclear Center, Bombay, India, en 1969, y, finalmente, una beca de la OEA para estudios en Biología Molecular en la Universidad de Chile (1970). La Prof. Beatriz Lizárraga consiguió una beca de la Organización de los Estados Americanos para un curso de Biología Molecular, seguido de una estadía de un año y medio en el laboratorio de Biología Molecular del Dr. Carlos Bacilio, en la Facultad de Ciencias de la Universidad de Chile. El magíster Álvaro Marcelo consiguió una posición en la Universidad de Temple, Philadelphia, en 1983, y el apoyo de la Comisión Fullbright para cubrir los pasajes. Permaneció varios años, hasta graduarse de $\mathrm{PhD}$ en Bioquímica, para regresar ulteriormente al Perú a reincorporarse a nuestro Centro. El magíster Mario Monteghirfo consiguió el apoyo de la Comisión Fullbright para una estadía de un año en investigaciones bioquímicas en la Commonwealth University, Virginia, Richmond (1983). A su retorno, se reincorporó a nuestra Institución y sus trabajos ulteriores, de gran calidad, lo califican como uno de los mejores investigadores del Centro. La profesora Liliana Ballivián, ya mencionada más arriba, consiguió una beca de la ICRO para una estadía de investigaciones en Biología Molecular en la Universidad Libre de Bruselas y otra beca para una estadía en el Laboratorio de Bioquímica y Biología Molecular de la Universidad de Bordeaux, Francia. La profesora Patricia Woll Tosso consiguió una beca para un curso sobre Estructura y Función de las Proteínas, de dos meses de duración, realizado en la Universidad de Concepción, Chile, en 1982, y otra beca de la UNESCO para un curso de un mes sobre Cultivo de Tejidos, realizado en Bulgaria, en 1986.

\section{Vuelta al sistema de Facultades}

Con el restablecimiento de la democracia en el Perú, la Universidad volvió al sistema de las Facultades y el Centro de Investigación de Bioquímica y Nutrición quedó nuevamente ligado a la Facultad de Medicina.

\section{Convenio con la Academia de Ciencias de la Unión Soviética}

A fines de 1983, recibimos la visita del Dr. Obchinikov, Vice-Presidente de la Academia de Ciencias de la Unión Soviética y Director del Instituto de Química Bioorgánica de Moscú; quedó muy bien impresionado de nuestro Centro de Investigación de Bioquímica y Nutrición y se acordó un convenio de cooperación entre nuestras instituciones. El Rector Gaston Pons Muzzo, yo y el Director de IVITA, Dr. Alberto Sato, fuimos invitados para visitar Moscú para la firma del convenio con nuestra Universidad, en 1984. El Rector no fue y solo viajamos el Dr. Sato, yo y el Dr. Ramiro Castro de la Mata, miembro del Concytec, quién firmó el convenio.

Para el acondicionamiento de un Laboratorio de Química Bioorgánica, se le asignó a la Academia de Ciencias de la Unión Soviética los sótanos de nuestro Centro. La Academia envió equipos, materiales y hasta muebles, por un valor aproximado de US $\$ 400$ 000,00. Una vez acondicionados los laboratorios, destacaron dos investigadores permanentes, que se turnaban anualmente para realizar investigaciones sobre principios activos de plantas medicinales. Nuestro Centro destacó algunos investigadores para colaborar con ellos. El año 1987, pedí mi cesantía de la Universidad, pero los soviéticos continuaron algunos años más.

El Centro de Investigación de Bioquímica y Nutrición, por la variedad y número de las investigaciones, la categoría de sus investigadores y la 
calidad de sus publicaciones se constituyó en uno de los centros de investigación más importantes de la Universidad Nacional Mayor de San Marcos y fue el Centro de Adiestramiento en el Perú del Proyecto Multinacional de Bioquímica de la Organización de los Estados Americanos, formando parte de los Centros de Excelencia de Bioquímica de Latinoamérica. Esta situación fue alcanzada por el esfuerzo conjunto y espíritu de superación de sus investigadores.

\section{El personal administrativo}

Debo hacer especial mención a la Srta. Margarita Ludeña, eficiente secretaria que trabajó conmigo durante todo el tiempo que duró mi función directiva. Los otros miembros del personal fueron los Srs. Maximiliano Ramos, Eulogio Flores, Domingo Valencia, Alberto Cabezas, Mariano Rojas, Rigoberto Collazos, Helí Cerna, Germán Pedroza, Juan de Dios Costarreal y Job Varela Morales, que cumplieron a satisfacción con las diversas tareas que les fueron asignadas.

En 1989, después del fallecimiento del Prof. Alberto Guzmán Barrón, la Universidad de San Marcos sacó una resolución, dándole su nombre al 'Centro de Investigación de Bioquímica y Nutrición: Alberto Guzmán Barrón', en reconocimiento a sus importantes servicios prestados.

\section{AGRADECIMIENTOS}

A la Mg. Inés Arnao Salas por la elaboración del resumen y abstract.

\section{REFERENCIAS BIBLIOGRÁFICAS}

1. Villavicencio M, Guzmán Barrón ES. Pathway of hexosa metabolism in lymphatic cells and lymphosarcoma. Fed Proc. $1955 ; 14: 1$.

2. Villavicencio M, Guzmán Barrón ES. Pathways of glucosa metabolism in lymphatic cells of rabbit's appendix and Gardners's mouse lymphosarcoma. Arch Biochem Biophys. 1957;67(1):121-37.

3. Guzmán Barrón ES, Villavicencio M, King DE Jr. $\mathrm{CO}_{2}$ fixation and utilization of ribose-5-phosphate by certain normal and tumor cells. Arch Biochem Biophys. $1955 ; 58(2): 500-2$.
4. Monge C, Palma R, Soto O, Loret de Mola A, Lastres J (Eds). Inauguración del Instituto de Bioquímica y Nutrición. An Fac Med. 1957;40(1):299-300.

5. Villavicencio M, Rosales F, Melgar E, Guerra R. Pathways of glucose metabolism in rabbit cerebral cortex. Acta Physiol Latinoam. 1959;8:219.

6. Villavicencio M, Rosales F, Guerra R. The metabolism of ribose-5-phosphate by mammalian tissues. Biochem Biophys Acta. 1961;53:495-508.

7. Villavicencio M, Guerra R, Rosales F. Oxidación de la glucosa en homogenizados de tejidos animales. Bol Soc Quim del Perú. 1963;29:55-8.

8. Goldman J, Rosales F, Villavicencio M, Guerra R. Pathways of glucose metabolism in rat submaxillary gland. Biochem Biophys Acta. 1964;82:303-12.

9. Cremona T, Kearney EB, Villavicencio M, Singer TP. Studies on the respiratory chain-linked dehydrogenase. V. Transformation of DPNH dehydrogenase to DPNHcytochrome $\mathrm{c}$ reductase and diaphorase under the influence of heat, proteolytic enzymes and urea. Biochem Z. 1963;338:407-42.

10. Villavicencio M, Neilands JB. An inducible ferrichromedegrading peptidase from Pseudomonas FC-1. Biochemistry. 1965;4(6):1092-7.

11. Goldthwait DA, Melgar E. Desoxyribonucleic acid nucleases. I. The use of a new method to observe the kinetics of deoxyribonucleic acid degradation by deoxyribonuclease I, deoxyribonuclease II, and Escherichia coli endonuclease I. J Biol Chem. 1968;243(17):4401-8.

12. Goldthwait DA, Melgar E. Deoxyribonucleic acid nucleases. II. The effects of metals on the mechanism of action of deoxyribonuclease I. J Biol Chem. 1968;243(17):440916.

13. Sakami W, Rosales F, Ritari SJ. Formation of the N5methyltetrahydrofolate-homocysteine methyltransferase holoenzyme from apoenzyme and adenosyl-B 12. Biochem Biophys Res Commun. 1970;40(2):271-6.

14. Guerra R, Melgar E, Villavicencio M. Alternative pathways of glucose metabolism in fetal rat brain. Biochem Biophys Acta. 1967;148(2):356-61.

15. Villavicencio $M$, Guerra R, Vásquez R. Oxidación de la glucosa en hígado de alpaca. Bol Soc Quim Perú. 1971;37:52.

16. Biscoglio de Jiménez Bonino M, Cascone O, Arnao AI, Santomé J, Sánchez D, Oré R, Villavicencio M. Isolation and characterization of alpaca growth hormone. Int J Peptide Prot Res. 1981;17(3):374-9.

17. Cascone O, Biscoglio de Jiménez Bonino $M$, Peña $C$, Santomé JA, Arnao I, Sánchez D, Oré R, Villavicencio M. Amino acid sequences around the cystine residues in alpaca growth hormone. Acta Physiol Pharmacol Latinoam. 1984;34(2):123-30.

18. Melgar E, Jouve H, Lizárraga B. A study of the binding of $\mathrm{Mn}^{2+}$ to bovine pancreatic deoxyribonuclease I and to deoxyribonucleic acid by electron paramagnetic resonance. Biol Chem. 1975;250(17):6631-5. 
19. Gil A, Melgar E, Lizárraga B, Sánchez-Romero, D. The role of $\mathrm{Ca}^{2+}$ on $\mathrm{pH}$-induced hydrodynamic changes of bovine pancreatic deoxyribonuclease A. J Biol Chem. 1978;253(9):3191-5.

20. Gil A, Bustamante C, Melgar E, Lizárraga, B. Multiple conformations of deoxyribonuclease A. Their separation at alkaline $\mathrm{pH}$ and low ionic strength in the presence of $\mathrm{Ca}^{2+}$. Biochim Biophys Acta. 1979; 579(2):298-302.

21. Haak H, Poma E, Soberón M. Mecanismo de acción de la fosfatasa ácida de hígado bovino. Bol Soc Quím Perú. 1989;55(4):209-19.

22. Haak H, Ochoa V, Guija E. Reactividad y función de los grupos sulfidrilos de la fosfatasa ácida de hígado bovino. Bol Soc Quim Perú. 1991;57(3):160-4.

23. Guzmán Barrón A, Blanco de Alvarado Ortiz T, Ayala Macedo G. Nutrición Humana. 1ra Ed. 1981. Ed. Perú Offset, Lima, Perú.

24. Ayala Macedo G, Dávila R, Tapia M. Nutrición y agricultura en comunidades campesinas de Puno. Primera parte. Lima, Perú: UNMSM-CIBN-Proyectos de Sistemas Agropecuarios Andinos, PISA. 1989.

25. Ramos Aliaga, R. \& Arroyave, G. Biochemical changes produced by cocaine in rats receiving different types of nutrition. Arch Latinoamer Nutr, 1969;19:69-79.
26. Ramos Aliaga, R \& Chiriboga, J. Enzymatic N-demethylation of cocaine and nutricional satatus. Arch Latinoamer Nutr; 1970;20:415-428.

27. Ramos Aliaga R \& Werner G. Increased activity of the mithochondrial cholesterol side-chain cleavage reaction in rats treated with cocaine. Res Comm in Substances of Abuse. 1983;4(3):267-70.

28. Ramos Aliaga R, Placencia M. Effect of cocaine intake on the development of fatty liver in rats fed a low protein diet. Arch Latinoamer Nutr, 1987;37(2):282-94.

Manuscrito recibido el 17 de octubre de 2007 y aceptado para publicación el 13 de diciembre de 2007.

Correspondencia:

Dr. Marino Villavicencio-Núñez

Centro de Investigación de Bioquímica y Nutrición.

Facultad de Medicina, UNMSM

Av. Grau 755. Lima 1, Perú

Correo-e: marinovillavicencio@yahoo.com.br 